\title{
Correlation between PD-L1 expression and clinicopathological characteristics of non-small cell lung cancer: A real-world study of a large Chinese cohort
}

\author{
Yan Jin ${ }^{1,2}$, Xuxia Shen ${ }^{1,2}$, Yunjian Pan ${ }^{2,3}$, Qiang Zheng ${ }^{1,2}$, Haiquan Chen ${ }^{2,3}$, Hong $\mathrm{Hu}^{2,3 \#}$, Yuan $\mathrm{Li}^{1,2 \#}$ \\ ${ }^{1}$ Department of Pathology, Fudan University Shanghai Cancer Center, Shanghai 200032, China; ${ }^{2}$ Department of Oncology, Shanghai Medical \\ College, Fudan University, Shanghai 200032, China; ${ }^{3}$ Department of Thoracic Surgery, Fudan University Shanghai Cancer Center, Shanghai \\ 200032, China \\ Contributions: (I) Conception and design: Y Li, H Hu; (II) Administrative support: H Chen; (III) Provision of study materials or patients: Y Jin, Y \\ Pan; (IV) Collection and assembly of data: Y Jin, X Shen; (V) Data analysis and interpretation: Y Jin, Q Zheng; (VI) Manuscript writing: All authors; \\ (VII) Final approval of manuscript: All authors. \\ "These authors contributed equally to this work. \\ Correspondence to: Yuan Li, MD, PhD. Department of Pathology, Fudan University Shanghai Cancer Center, 270 Dong-An Road, Shanghai 200032, \\ China. Email: whliyuan@hotmail.com; Hong Hu, MD, PhD. Department of Thoracic Surgery, Fudan University Shanghai Cancer Center, 270 \\ Dong-An Road, Shanghai 200032, China. Email: huhong0997@163.com.
}

Background: Programmed death ligand-1 (PD-L1) is a predictive marker of anti-programmed death protein 1 (PD-1)/PD-L1 therapies for non-small cell lung cancer (NSCLC). However, little is known between PD-L1 expression and the clinicopathological characteristics of NSCLC in the Chinese population in a real-world setting.

Methods: We analyzed PD-L1 expression by immunohistochemistry (IHC) in NSCLC patients using the $22 \mathrm{C} 3$ clone on the Dako Autostainer Link 48 platform. We then examined the associations of PD-L1 expression with clinicopathological characteristics, stromal tumor-infiltrating lymphocytes (TILs) and major molecular features.

Results: A total of 1,156 recently NSCLC specimens including 827 sequentially resected specimens and 293 biopsy specimens were enrolled in our study. PD-L1 high expression was observed in $9.7 \%$ of 827 NSCLC patients, including $6.5 \%$ with adenocarcinoma (ADC, $n=690$ ), and $27.4 \%$ with squamous cell carcinoma (SqCC, $\mathrm{n}=117)$. These results showed higher expression rates than those in archived samples ( $>5$ years old, $\mathrm{n}=329)$, that were previously reported by our group $(4.9 \%, 0.5 \%$, and $13.9 \%$ in NSCLC, $\mathrm{ADC}$, and SqCC, respectively). The prevalence of PD-L1 expression was lower in surgical resection samples than in small biopsy samples. PD-L1 high expression in the lung biopsy was less likely present in the primary cancer than in metastases, and was also associated with a high level of stromal TILs $(\mathrm{P}=0.029)$ and PD-L1positive immune cells $(\mathrm{IC})(\mathrm{P}<0.001)$. Both $\mathrm{PD}-\mathrm{L} 1$ high and low expressions were more frequent in EGFRwild type than in mutant type $(\mathrm{P}<0.001)$.

Conclusions: This study demonstrates that expression of PD-L1 is linked to the type of tumor specimens, resection versus biopsy specimens, and biopsies of primary versus metastatic cancers. These findings have substantial implications for clinical practice.

Keywords: Programmed death-ligand 1 (PD-L1); non-small cell lung cancer (NSCLC); immunohistochemistry (IHC)

Submitted Oct 16, 2019. Accepted for publication Oct 21, 2019.

doi: $10.21037 /$ jtd.2019.10.80

View this article at: http://dx.doi.org/10.21037/jtd.2019.10.80 


\section{Introduction}

The approach to the diagnosis and molecular analysis of non-small cell lung cancer (NSCLC) has advanced significantly in the last decade. There is a revolution driven by the development of drugs against particular sub-groups of tumor defined by their genetic pathology or protein expression $(1,2)$. The remarkable success of therapies with antibodies that block the interaction of programmed death protein 1 (PD-1) and programmed death ligand 1 (PD-L1) introduced the era of immunotherapy for advanced NSCLC (3-5).

The KEYNOTE trials reported that pembrolizumab prolonged the overall survival (OS) rates of NSCLC patients with PD-L1 immunohistochemistry (IHC) positive expression (at least $1 \%$ of tumor cells, TCs) (5-7). Pembrolizumab was approved for the first line treatment of patients with metastatic NSCLC whose tumors demonstrate PD-L1 (22C3, Dako platform) staining in $\geq 50 \%$ of TCs, or patients with locally advanced and/or metastatic NSCLC with prior chemotherapy and a $\geq 1 \%$ of TCs. Hence, the association between PD-L1 expression and clinicopathological characteristics has become an important consideration. A number of investigators have already presented that $\mathrm{PD}-\mathrm{L} 1$ positive rates range from $13 \%-35 \%$ in archived NSCLC samples (8-11). However, little is known between PD-L1 expression and the clinicopathological characteristics of NSCLC in the Chinese population in a real-world setting.

Immune cells (ICs) in the lung cancer microenvironment primarily comprise $\mathrm{T}$ cells, macrophages, and mast cells. They are generally found at the invasive tumor margin (12). Recently, several studies reported that a high number of tumor-infiltrating lymphocytes (TILs) were generally associated with a good outcome in NSCLC patients $(13,14)$. Donnem et al. focused on the prognostic impact of TILs on immunoscore assessment and TNM-immunity. Although the implication of TILs has been understood to some extent, these studies neither assessed PD-L1 expression on ICs nor described the relationship between stromal TILs and PD-L1 expression.

The aim of this study was to provide real-world data on PD-L1 protein expression in surgically resected and biopsied NSCLC samples and to compare recently resected with archived tumor samples. We also analyzed the correlation between PD-L1 expression and clinicopathological characteristics, stromal TILs, and pulmonary major driver genes alterations in Chinese NSCLC patients.

\section{Methods}

\section{Patients and samples}

Tumor samples were collected from 1,156 NSCLC patients who underwent sequentially surgical resection and 293 biopsy specimens in our institution between September 2017 and June 2018. Clinical data, PD-L1 expression data, and molecular alteration data were retrieved from the patients' medical records. Patients who received neoadjuvant chemotherapy and had a history of other malignant tumors were excluded. Patients were classified according to the smoking status as never-smokers ( $<100$ lifetime cigarettes) and smokers. The histology of the lung cancers was classified according to the 2015 WHO classification (15). The tumor pathologic stage was characterized using the AJCC staging system (8th Edition) (16). The Institutional Review Board (IRB) of Fudan University Shanghai Cancer Center had approved this study.

\section{Analysis of PD-L1 protein expression by IHC}

PD-L1 protein staining was performed on formalin-fixed paraffin-embedded (FFPE) sections ( $4 \mu \mathrm{m}$ thick) by IHC immediately after the operation. In specimens whose the maximum diameter was greater than $1 \mathrm{~cm}$, a representative slide was selected for staining. The representative slide was defined as a section contained the most diverse histological subtypes. This assay was performed on the Dako Autostainer Link 48 platform with an automated staining protocol using a mouse monoclonal anti-PD-L1 antibody (22C3). PD-L1 expression was evaluated by the tumor proportion score (TPS), which is defined as the percentage of PD-L1-positive TCs over total TCs. The evaluation of the score included partial or complete membranous staining (at least $1+$ intensity). All other cells, such as tumorassociated ICs, normal/non-neoplastic cells, and necrotic cells, were excluded from the evaluation. PD-L1 expression in TCs was classified into three levels: negative expression (TPS $<1 \%$ ), low expression (TPS 1-49\%), and high expression (TPS $\geq 50 \%$ ). Furthermore, we evaluated PDL1 expression in ICs. PD-L1 ICs were defined as positive when there were more than $1 \%$ positive mononuclear cells (including lymphocytes and plasma cells) in tumor stroma, according to the atlas of the PD-L1 SP142 assay by IHC testing in lung cancer (17). The results were interpreted by using light microscope (Olympus BX43, Japan) by two pathologists who were blinded to clinical data and patient outcomes (Y. L. and Y. J). 


\section{Evaluation of stromal TILs}

The scoring of stromal TILs was performed on hematoxylin \& eosin (H\&E)-stained FFPE tissue sections, as previously described (18). We evaluated stromal TILs which often localize to surgical margins, including lymphocytes and plasma cells. The stromal TILs were assessed in multiple stromal regions and not only in hot spots. The percentage of tumor stromal containing ICs was classified as low stromal TILs (1-49\%), and high stromal TILs ( $\geq 50 \%)$.

\section{Status of other driver genes}

EGFR mutations within exons 18 to 21 in FFPE tissues were examined using the ADx-ARMS (amplification refractory mutation system) kit (Amoy Diagnostics, Xiamen, China), as described previously (8). The $A L K$ rearrangement was analyzed by fluorescence in situ hybridization (FISH) using a Vysis break-apart rearrangement probe (Abbott Diagnostics, Abbott Park, IL, USA) or IHC (clone D5F3,
VMSI) on FFPE tumors.

\section{Statistical analysis}

Statistical analyses were performed using the software package Statistical Package for Social Sciences, version 20.0, for Windows (SPSS, Chicago, IL, USA). The chisquare test or Fisher's exact test was used to determine potential associations. All statistical values were determined using two-tailed statistical analyses, and $\mathrm{P}$ values $<0.05$ were considered to indicate a statistically significant difference.

\section{Results}

\section{Patient and tumor characteristics}

The surgical resection group consisted of 827 recently resected and 329 archived ( $>5$ years old) NSCLC samples (Table 1). The archived tissues have been previously described (8). The current acquired resection tumor

Table 1 Clinicopathological features of patients with non-small cell lung cancer (NSCLC)

\begin{tabular}{|c|c|c|c|c|}
\hline Variables & Archival samples & \multicolumn{2}{|c|}{ Recent samples } & $P$ value \\
\hline Total & 329 & 827 & 293 & \\
\hline Male & 225 & 421 & 216 & \\
\hline Female & 104 & 406 & 77 & \\
\hline Median & 61 & 61 & 64 & \\
\hline Range & $27-83$ & $17-83$ & $27-75$ & \\
\hline Histology & & & & $<0.001$ \\
\hline Adenocarcinoma (ADC) & 221 & 690 & 211 & \\
\hline Adenosquamous carcinoma (ASC) & 0 & 6 & 2 & \\
\hline Large cell carcinoma (LCC) & 0 & 2 & 2 & \\
\hline Pleomorphic carcinoma (PC) & 0 & 2 & 2 & \\
\hline Atypical carcinoid tumor (AC) & 0 & 1 & 0 & \\
\hline PD-L1 expression & & & & 0.001 \\
\hline Negative & $283(86.0 \%)$ & $572(69.2 \%)$ & $94(32.1 \%)$ & \\
\hline Low expression & $30(9.1 \%)$ & $175(21.2 \%)$ & $74(25.3 \%)$ & \\
\hline High expression & $16(4.9 \%)$ & $80(9.7 \%)$ & $125(42.7 \%)$ & $<0.001$ \\
\hline
\end{tabular}



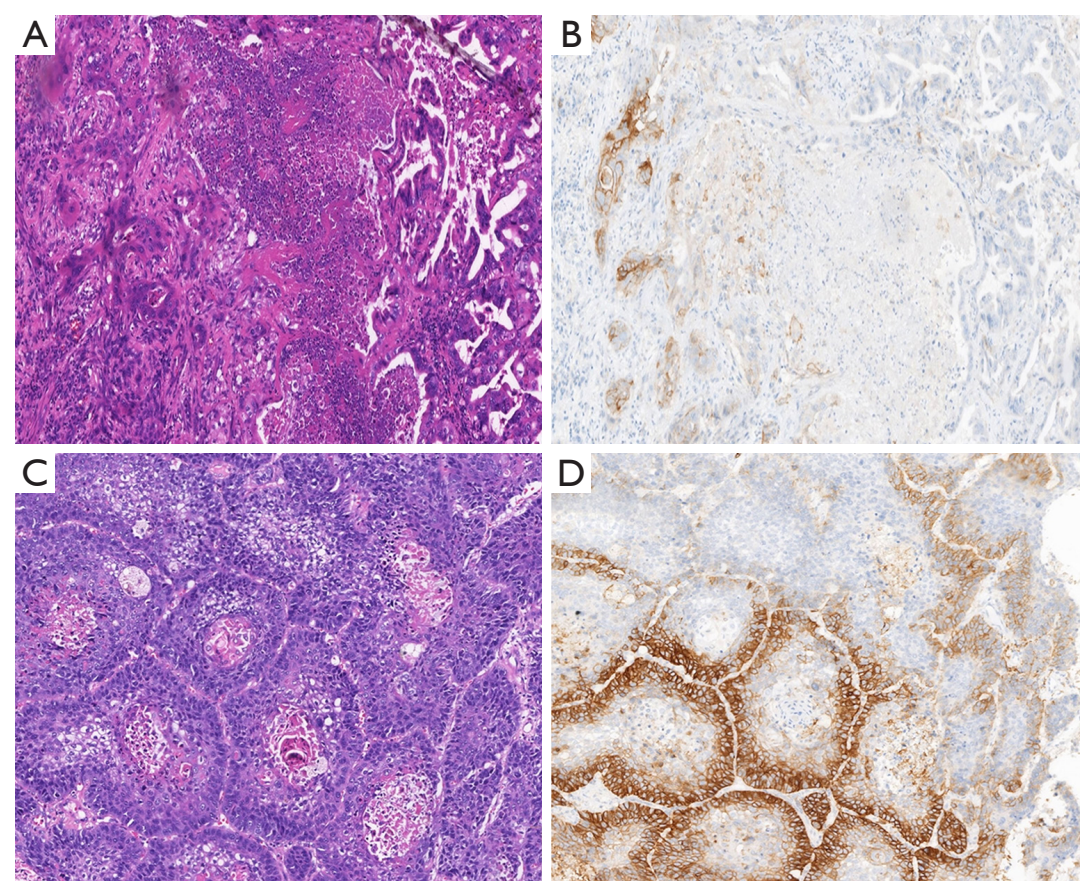

Figure 1 Representative images of programmed death ligand-1 (PD-L1) immunostaining in adenocarcinoma and squamous cell carcinoma ( $\times 200)$. (A) and (B) show hematoxylin and eosin (H\&E) staining and the membranous staining of PD-L1 expression in adenocarcinoma; (C) and (D) show H\&E staining and PD-L1 expression in squamous cell carcinoma. PD-L1 expression in tumor cells showed different staining patterns based on tumor differentiation. In the same specimens, a well-differentiated area shows low PD-L1 expression, whereas a poorly differentiated area shows strong PD-L1 expression.

samples included those from 406 women $(49.1 \%)$ and 421 men $(50.9 \%)$, with a median age of 61 years (range, $17-83$ years). Of the 827 patients, 520 (62.9\%) were non-smokers, and 307 were smokers. The histological characterization of tumors revealed that 690 samples were adenocarcinoma (ADC, 83.4\%), 117 were squamous cell carcinoma (SqCC, 14.1\%), and 20 were other histologic type NSCLC $(2.4 \%$; 9 large cell neuroendocrine carcinomas, 6 adenosquamous carcinomas, 2 large cell carcinomas, 2 pleomorphic carcinomas, and 1 atypical carcinoid tumor). Of the 827 patients, characterization of the pathological stage of each tumor revealed 606 patients in stage I, 100 patients in II, 114 patients in III, and 7 patients in IV.

A total of 293 small biopsy cases included 244 primary pulmonary specimens and 49 metastatic specimens that involved lymphocytes, the brain, bone, soft tissue, adrenal gland, liver, bronchus, ovary, and pleura. Of these patients, $216(73.7 \%)$ were male and $77(26.31 \%)$ were female. The median age was 64 years (range, $27-75$ years) (Table 1).

\section{PD-L1 protein expression in NSCLC}

PD-L1 protein expression in TCs was defined as complete circumferential or partial cell membrane staining (Figure 1). Among the NSCLC patients, 80 (9.7\%) had high PD-L1 expression, and 175 (21.1\%) had low PD-L1 expression. High PD-L1 expression was significantly more common in SqCC than in non-squamous NSCLC (ADC and other histologic type NSCLC) (SqCC 27.4\% vs. ADC 6.5\% vs. other histologic type NSCLC $15 \%, \mathrm{P}<0.001$ ) (Figure 2).

Regarding ADC, PD-L1 high expression and low expression were observed in $6.5 \%$ and $17.7 \%$ of patients, respectively (Figure 2). PD-L1 high expression was significantly associated with female sex $(\mathrm{P}=0.002)$, a large tumor size $(\mathrm{P}<0.001)$, pleural invasion $(\mathrm{P}=0.001)$, venous and lymphatic invasion $(\mathrm{P}<0.001)$, smoking history $(\mathrm{P}<0.001)$, and advanced pathological stage $(\mathrm{P}<0.001)$ (Table 2$)$. The ADC subtype groups with less frequent PD-L1 expression included the papillary $(6 / 163,3.7 \%)$, and acinar $(13 / 352,3.7 \%)$. The subtype groups with the more frequent 

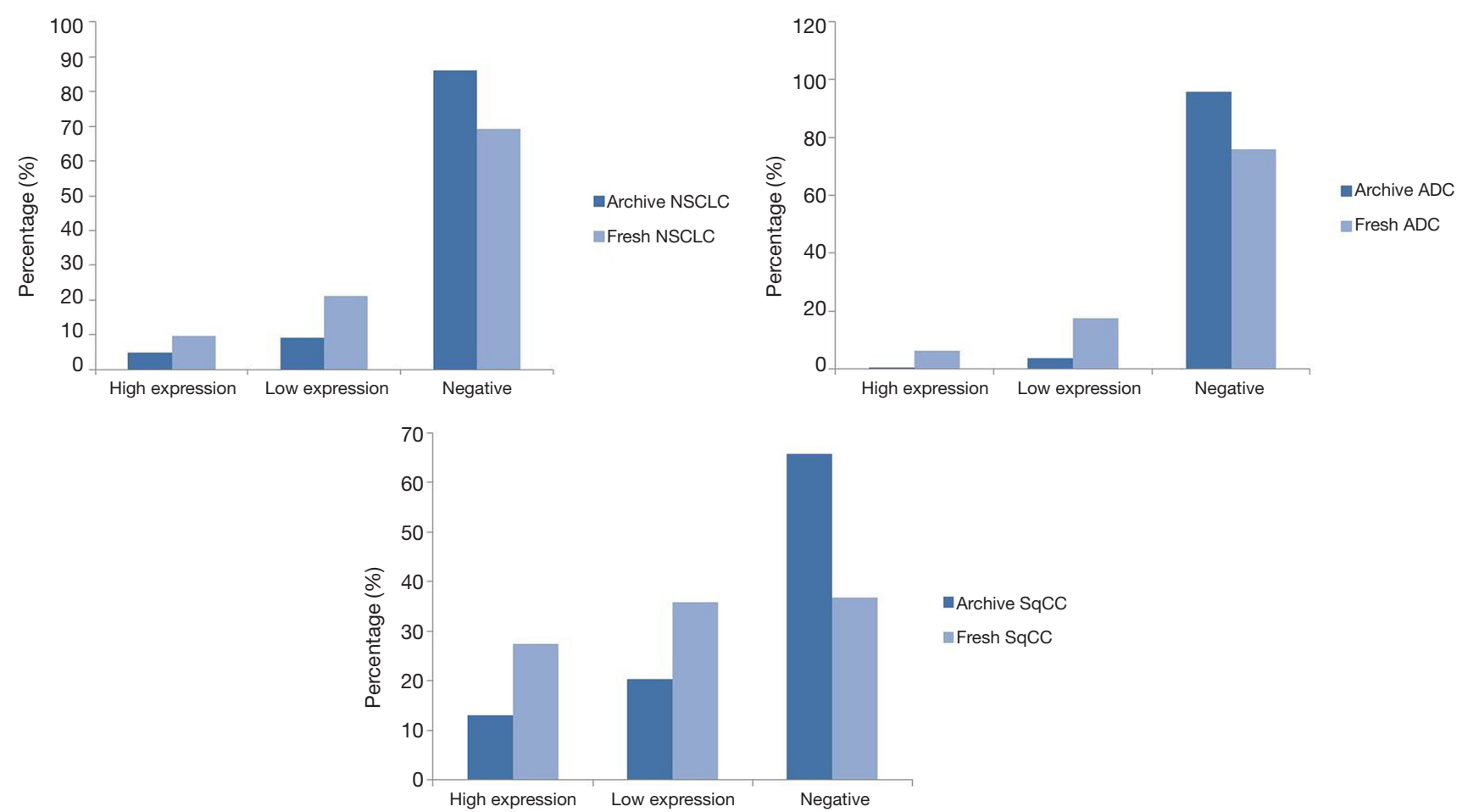

Figure 2 Comparison of PD-L1 expression in recently acquired samples and archived NSCLC samples. PD-L1, programmed death ligand-1; NSCLC, non-small cell lung cancer; ADC, adenocarcinoma; SqCC, squamous cell carcinoma.

PD-L1 expression included the solid $(22 / 63,34.9 \%)$ and micropapillary $(4 / 20,20.0 \%)$ subtypes $(\mathrm{P}<0.001)$.

In the SqCC subgroup, the PD-L1 high expression, low expression and negative expression were observed in $27.4 \%, 35.8 \%$, and $36.8 \%$ of patients, respectively. PD-L1 high expression was significantly associated with the nonkeratinizing type $(\mathrm{P}=0.012)$ and the absence of perineural invasion $(\mathrm{P}=0.001)$ (Table 3).

PD-L1 high expression was observed in $15 \%$ of the remaining patients (3/20, 2 adenosquamous carcinomas, 1 large cell carcinoma). The PD-L1 low expression was observed in $55 \%$ of the remaining patients (11/20, 5 large cell neuroendocrine carcinomas, 3 adenosquamous carcinomas, 2 pleomorphic carcinomas, and 1 atypical carcinoid tumor). Four large cell neuroendocrine carcinomas, 1 adenosquamous carcinoma, and 1 large cell carcinoma showed negative PD-L1 expression.

We subsequently evaluated the prevalence of PDL1 expression in small biopsy specimens. Among the 244 primary pulmonary small biopsy specimens, PD-L1 high and low expression were found in $76(31.1 \%)$ and $63(25.8 \%)$ patients. In the 49 metastasis specimens, PD-L1 high expression and low expression were observed in 18 (36.7\%) and $11(22.4 \%)$ patients, respectively.

\section{Correlation of PD-L1 expression and the tumor microenvironment in NSCLC}

A total of 368 NSCLC patients were examined to define whether PD-L1 expression correlated with the tumor stromal immune microenvironment (Figure 3). High and low stromal TILs were observed in $44(12.0 \%)$ and $324(88.0 \%)$ tumors, respectively. Patients with high stromal TILs were found to exhibit higher PD-L1 protein expression than those with low stromal TILs ( $\mathrm{P}=0.029$, Table 4). A high stromal TIL density was correlated with clinicopathological parameters, although the difference was not significant. On the other hand, PD-L1 high expression was significantly associated with the PD-L1 IC-positive status $(\mathrm{P}<0.001$, Table 4).

\section{Association between PD-L1 expression and the status of major driver genes}

A total of 542 NSCLC patients were available to study $E G F R$ mutations: 156 patients had wild-type $E G F R$, 
Table 2 Clinicopathological features of patients with adenocarcinoma

\begin{tabular}{|c|c|c|c|c|c|}
\hline Variables & Total $(n=690)$ & \multicolumn{3}{|c|}{ PD-L1 expression, n (\%) } & $P$ value \\
\hline Sex & & & & & 0.002 \\
\hline Male & 393 & $315(80.2)$ & $62(15.8)$ & $16(4.1)$ & \\
\hline Female & 297 & $208(70.0)$ & $60(20.2)$ & $29(9.8)$ & \\
\hline$<61$ years & 339 & $267(78.8)$ & $53(15.6)$ & $19(5.6)$ & \\
\hline$\geq 61$ years & 351 & 256 (72.9) & $69(19.7)$ & $26(7.4)$ & \\
\hline Tumor size & & & & & $<0.001$ \\
\hline$<2 \mathrm{~cm}$ & 341 & $286(83.9)$ & $44(12.9)$ & $11(3.2)$ & \\
\hline Lepidic & 56 & $52(92.2)$ & $4(7.1)$ & $0(0.0)$ & \\
\hline Acinar & 352 & $278(79.0)$ & $61(17.3)$ & $13(3.7)$ & \\
\hline Papillary & 163 & $128(78.5)$ & $29(17.8)$ & $6(3.7)$ & \\
\hline Solid & 63 & $20(31.7)$ & $21(33.3)$ & $22(34.9)$ & \\
\hline Micropapillary & 20 & $9(45.0)$ & $7(35.0)$ & $4(20.0)$ & \\
\hline Pleural invasion & & & & & 0.001 \\
\hline Absent & 587 & $460(78.4)$ & $92(15.7)$ & $35(6.0)$ & \\
\hline Visceral invasion & 103 & $63(61.2)$ & $30(29.1)$ & $10(9.7)$ & \\
\hline Present & 10 & $5(50.0)$ & $3(30.0)$ & $2(20.0)$ & \\
\hline Smoking & & & & & $<0.001$ \\
\hline No & 483 & $385(79.7)$ & $76(15.7)$ & $22(4.6)$ & \\
\hline Yes & 207 & $138(66.7)$ & $46(22.2)$ & $23(11.1)$ & \\
\hline Stage & & & & & $<0.001$ \\
\hline Early stage (I and II) & 602 & $478(79.4)$ & $91(15.1)$ & $33(5.5)$ & \\
\hline Advanced stage (III and IV) & 88 & $45(51.1)$ & $31(35.2)$ & $12(13.6)$ & \\
\hline
\end{tabular}

\footnotetext{
\#, invasive adenocarcinoma.
}

384 had mutant $E G F R$, and 1 had a gene amplification. Histologically, the majority of tumor specimens were ADC (94.6\%). PD-L1 high and low expression was more frequent in the EGFR wild-type group than in the mutant group $(\mathrm{P}<0.001)$ (Table 5). There was a significant association between the EGFR mutation types and the $\mathrm{PD}$ L1 expression level. Rare EGFR mutations (exon 18 and 20 mutations, double mutations) demonstrated higher 
Table 3 Clinicopathological features of patients with squamous cell carcinoma

\begin{tabular}{|c|c|c|c|c|c|}
\hline Variables & Total $(n=117)$ & \multicolumn{3}{|c|}{ PD-L1 expression, $\mathrm{n}(\%)$} & $P$ value \\
\hline Sex & & & & & 0.110 \\
\hline Male & 108 & $42(38.9)$ & $36(33.3)$ & $30(27.8)$ & \\
\hline Female & 9 & $1(11.1)$ & $6(66.7)$ & $2(22.2)$ & \\
\hline$\geq 65$ years & 61 & $25(41.0)$ & $19(31.1)$ & $17(27.9)$ & \\
\hline Tumor size & & & & & 0.273 \\
\hline$<3.5 \mathrm{~cm}$ & 10 & $6(60.0)$ & $2(20.0)$ & $2(20.0)$ & \\
\hline Non-keratinizing & 101 & $32(31.7)$ & $38(37.6)$ & $31(30.7)$ & \\
\hline Pleural invasion & & & & & 0.124 \\
\hline Absent & 100 & $33(33.0)$ & $38(38.0)$ & $29(29.0)$ & \\
\hline Visceral invasion & 17 & $10(58.8)$ & $4(23.5)$ & $3(17.6)$ & \\
\hline Venous and lymphatic invasion & & & & & 0.859 \\
\hline Absent & 84 & $32(38.1)$ & $29(34.5)$ & $23(27.4)$ & \\
\hline Present & 33 & $11(33.3)$ & $13(39.4)$ & $9(27.3)$ & \\
\hline Perineural invasion & & & & & 0.001 \\
\hline Stage & & & & & 0.315 \\
\hline Early stage (I and II) & 88 & $32(36.4)$ & $29(33.0)$ & $27(30.7)$ & \\
\hline Advanced stage (III and IV) & 29 & $11(37.9)$ & $13(44.8)$ & $5(17.2)$ & \\
\hline
\end{tabular}

expression rates than classical mutations (exon 19 and 21 mutations).

In addition, we examined the relationship between the $A L K$ gene rearrangement and PD-L1 expression in 320 resected NSCLCs. PD-L1 high expression and low expression were observed in $6.9 \%(20 / 289)$ and $19.0 \%$ (55/289) of $A L K$-negative patients, respectively. For the $A L K$ gene rearrangement group, PD-L1 high expression and low expression were observed in $6.5 \%(2 / 31)$ and $32.3 \%(10 / 31)$ patients, respectively. PD-L1 expression was not associated with the $A L K$ gene rearrangement $(\mathrm{P}=0.218)$.

\section{Discussion}

To our knowledge, this study represents the largest comprehensive study of PD-L1 expression with clinicopathological characteristics in Chinese patients with NSCLC. Here, we show the following: (I) the prevalence of PD-L1 high expression $(27.4 \%)$ in the surgically resected SqCC population was higher than that in the ADC population (6.5\%), which is in accordance with previous reports $(8,11)$; (II) surgically resected tumor samples showed lower rates of PD-L1 expression 

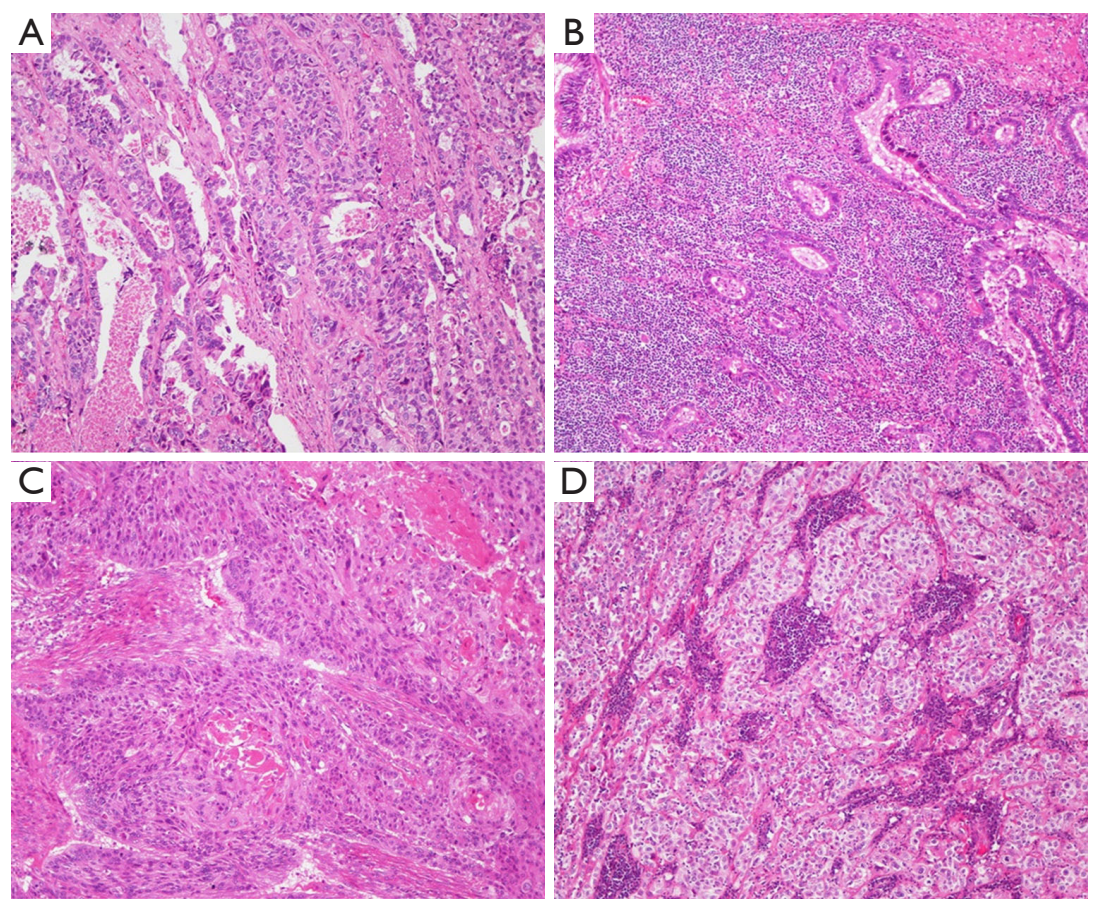

Figure 3 Representative H\&E staining images of stromal tumor-infiltrating lymphocytes (TILs) ( $\times 200)$. (A) and (B) show a negative ( $<50 \%)$, and positive ( $\geq 50 \%)$ stromal TILs status in adenocarcinoma, respectively; (C) and (D) show a negative $(<50 \%)$, and positive ( $\geq 50 \%)$ stromal TILs status in squamous cell carcinoma, respectively.

Table 4 Correlation of PD-L1 expression and tumor microenvironment status

\begin{tabular}{|c|c|c|c|c|c|}
\hline \multirow{2}{*}{ Variables } & \multirow{2}{*}{ Total $(n=368)$} & \multicolumn{3}{|c|}{ PD-L1 expression, $n(\%)$} & \multirow{2}{*}{$P$ value } \\
\hline & & Negative & Low expression & High expression & \\
\hline sTILs status & & & & & 0.029 \\
\hline High $(<50 \%)$ & 324 & $215(66.4)$ & $73(22.5)$ & $36(11.1)$ & \\
\hline Low ( $\geq 50 \%)$ & 44 & $21(47.7)$ & $13(29.5)$ & $10(22.7)$ & \\
\hline ICs status & & & & & $<0.001$ \\
\hline Negative $(\geq 1 \%)$ & 108 & $46(42.6)$ & $31(28.7)$ & $31(28.7)$ & \\
\hline
\end{tabular}

PD-L1, programmed death ligand-1; sTILs, stromal tumor-infiltrating lymphocytes; IC, immune cells.

than small biopsy samples; high PD-L1 expression of the lung biopsy was less likely present in the primary cancer than in metastases; (III) in ADC, PD-L1 high expression was more likely to appear among the solid and micropapillary subtypes; in SqCC, PD-L1 high expression was significantly associated with the non-keratinizing type; (IV) PD-L1 TCs high expression was significantly associated with high stromal TILs $(\geq 50 \%)$ and the PDL1 IC-positive status; (V) PD-L1 high expression was significantly associated with no EGFR mutation.

Previously reported tumor PD-L1 positivity detected by the $22 \mathrm{C} 3$ assay varied widely $(5-7,10,11)$. Several studies consistently reported that high PD-L1 expression was more frequent in SqCC than in ADC. One key finding of our study was that PD-L1 expression in recently resected tumor samples was higher than that in archived samples. In this study, the PD-L1 positive rates were similar to those reported in previous studies. However, the prevalence of PD-L1 in 
Table 5 Correlation of programmed death ligand-1 (PD-L1) expression and EGFR mutation

\begin{tabular}{|c|c|c|c|c|c|}
\hline EGFR mutation & Total $(n=541)$ & \multicolumn{3}{|c|}{ PD-L1 expression, $\mathrm{n}(\%)$} & $P$ value \\
\hline Mutations & & & & & $<0.001$ \\
\hline Rare mutation & 45 & $34(75.6)$ & $6(13.3)$ & $5(11.1)$ & \\
\hline Exon 21 mutation & 195 & $165(84.6)$ & $25(12.8)$ & $5(2.6)$ & \\
\hline
\end{tabular}

fresh tumor samples was conspicuously higher than that in archived samples (8). Our previous study assessed archived ( $>5$ years old) tumor samples (Figure $2 A$ ). Recently, one study reported that the concordance of archived samples $<3$ years old was the highest, among recently acquired samples (19). To our knowledge, protein expression can be influenced by the ageing of FFPE tissue blocks (20). It appears that the PDL1 protein may decay under different storage conditions. Our data suggest that $\mathrm{PD}-\mathrm{L} 1$ protein expression should ideally be determined immediately after the excision of primary lesions.

Another key finding of our study is the heterogeneity of PD-L1 expression in NSCLC. We identified a highly significant relationship between the histologic patterns of ADC and PD-L1 expression. Poorly differentiated histologic patterns, such as solid and micropapillary patterns were much more likely to express PD-L1, and well or moderately differentiated lepidic, acinar, and papillary patterns were much less likely to express PD-L1. Interestingly, in the same specimens, the well-differentiated area exhibited low PD-L1 expression intensity, whereas the poorly differentiated area exhibited strong PD-L1 expression intensity (Figure 1). Similar to ADC, PD-L1 expression was also prevalent in poorly differentiated SqCC (Figure 1). Recently, several studies have also reported that the poor differentiation histology was associated with high PD-L1 expression $(8,21,22)$. Considering the potential difference in PD-L1 expression, we should choose a representative section that contained the most diverse histological subtypes and then take an average value of PDL1 expression. These findings highlight the importance of histologic growth patterns when comparing the prevalence of PD-L1 positivity across studies.

In our study, PD-L1 high expression was observed in $29.3 \%$ of NSCLC biopsy specimens, which was higher than in surgically resected specimens (9.7\%). Moreover, interestingly, we found that high PD-L1 expression in the lung biopsy showed lower rates than those in the metastatic sites. One prior study reported that prevalence of PD-L1 TPS $\geq 50 \%$ and PD-L1 TPS $\geq 1 \%$ was similar across geographic regions, between surgical specimens and biopsies, and irrespective of whether tissue was from the primary tumor or from metastases (23). Such a discrepancy could be the result of tumor heterogeneity and different clinical stages. It is evident that PD-L1 expression in small biopsy samples might not represent that of the entire tumor specimen because of cancer heterogeneity. The mechanism of intra-tumoral PD-L1 expression heterogeneity is well understood (24-26). Furthermore, most biopsy specimens were obtained from patients with advanced lung cancer. In contrast, the majority of excision specimens were operable in early-stage tumors. These results demonstrate that PDL1 expression may reveal heterogeneity, and it is important to understand the effect of different sample sites on PD-L1 expression levels to assess their suitability for testing (27). Further work is needed to understand the mechanisms of intra- and inter-tumoral heterogeneity for clinical treatment guidance of patients with advanced unresectable lung cancers.

Recently, studies have indicated a trend that patients suitable for immunotherapy often lack known driver mutations $(11,28,29)$. We also showed that PD-L1 positive expression was more frequent in the EGFR wild type group than in the mutation group. However, some (4.4\%) patients with the EGFR mutation still expressed PD-L1; thus, more attention needs to be paid to these patients.

Some studies have revealed that TILs, especially T cells, have a major impact on the clinical course of several cancers, including melanoma and lung, melanoma, head and neck, breast, bladder, urothelial, ovarian, colorectal, renal, and prostate cancers $(12,13)$. Approximately $80 \%$ 
of lymphocytes are $\mathrm{T}$ cells expressing various activation antigens. Most prior studies evaluating TILs in lung cancer applied IHC (CD4, CD8, and CD3) to differentiate IC subsets and to assess their density, distribution and localization $(21,30)$. Here, we evaluated stromal TILs localized only in the invasive margin by commonly used H\&E staining methods. We found a significant correlation between PD-L1 expression and stromal TIL levels. Furthermore, our results showed that high PD-L1 expression was significantly associated with the presence of PD-L1-positive ICs. Further study should be carried out to accurately determine the relationship between PDL1 expression and ICs by IHC or other new technologies. The limitations of this study include the lack of an accurate evaluation of stromal TILs and a short follow-up time, which was not sufficient to compare differences in OS and disease-free survival (DFS). Nevertheless, this is the first correlation analysis of PD-L1 expression and stromal ICs.

In conclusion, our results revealed that PD-L1 higher expressing patterns of ADC and non-keratinizing SqCC as well as comparison with different metastatic expression. For patients with recurrence and metastasis, a re-biopsy is strongly suggested to evaluate the status of PD-L1. Only when sufficient fresh tissue cannot be obtained by a biopsy for various clinical conditions should archived blocks be considered as a complementary method. Our data also indicate that the $\mathrm{PD}-\mathrm{L} 1$ protein may decay under poor storage conditions. To ensure the reliability and authenticity of the expression level of PD-L1, IHC should be performed as soon as possible on postoperative specimens.

\section{Acknowledgments}

Funding: This study was supported by the National Nature Science Foundation of China (grant number 81472173 and 81972171).

\section{Footnote}

Conflicts of Interest: The authors have no conflicts of interest to declare.

Ethical Statement: The authors are accountable for all aspects of the work in ensuring that questions related to the accuracy or integrity of any part of the work are appropriately investigated and resolved. This study was approved by the institutional review board of the Shanghai Cancer Center, Fudan University, Shanghai, China (IRB,
No. IRB\# 090977-1). All procedures in studies that involved human participants were performed in accordance with the ethical standards of the institutional and/or national research committee and with the 1964 Declaration of Helsinki and its later amendments or with comparable ethical standards.

\section{References}

1. Scarpa M, Kotsafti A, Fassan M, et al. Immunonutrition before esophagectomy: Impact on immune surveillance mechanisms. Tumour Biol 2017;39:1010428317728683.

2. Dolled-Filhart M, Roach C, Toland G, et al. Development of a Companion Diagnostic for Pembrolizumab in NonSmall Cell Lung Cancer Using Immunohistochemistry for Programmed Death Ligand-1. Arch Pathol Lab Med 2016. [Epub ahead of print].

3. Shukuya T, Carbone DP. Predictive Markers for the Efficacy of Anti-PD-1/PD-L1 Antibodies in Lung Cancer. J Thorac Oncol 2016;11:976-88.

4. Taube JM, Galon J, Sholl LM, et al. Implications of the tumor immune microenvironment for staging and therapeutics. Mod Pathol 2018;31:214-34.

5. Garon EB, Rizvi NA, Hui R, et al. Pembrolizumab for the treatment of non-small-cell lung cancer. N Engl J Med 2015;372:2018-28.

6. Herbst RS, Baas P, Kim DW, et al. Pembrolizumab versus docetaxel for previously treated, PD-L1-positive, advanced non-small-cell lung cancer (KEYNOTE-010): a randomised controlled trial. Lancet 2016;387:1540-50.

7. Reck M, Rodriguez-Abreu D, Robinson AG, et al. Pembrolizumab versus Chemotherapy for PD-L1Positive Non-Small-Cell Lung Cancer. N Engl J Med 2016;375:1823-33.

8. Pan $\mathrm{Y}$, Zheng D, Li Y, et al. Unique distribution of programmed death ligand 1 (PD-L1) expression in East Asian non-small cell lung cancer. J Thorac Dis 2017;9:2579-86.

9. Lin G, Fan X, Zhu W, et al. Prognostic significance of PD-L1 expression and tumor infiltrating lymphocyte in surgically resectable non-small cell lung cancer. Oncotarget 2017;8:83986-94.

10. Sun JM, Zhou W, Choi YL, et al. Prognostic Significance of PD-L1 in Patients with Non-Small Cell Lung Cancer: A Large Cohort Study of Surgically Resected Cases. J Thorac Oncol 2016;11:1003-11.

11. Evans M, O'Sullivan B, Hughes F, et al. The Clinicopathological and Molecular Associations of PD-L1 
Expression in Non-small Cell Lung Cancer: Analysis of a Series of 10,005 Cases Tested with the 22C3 Assay. Pathol Oncol Res 2018. [Epub ahead of print].

12. Bremnes RM, Busund LT, Kilvaer TL, et al. The Role of Tumor-Infiltrating Lymphocytes in Development, Progression, and Prognosis of Non-Small Cell Lung Cancer. J Thorac Oncol 2016;11:789-800.

13. Fridman WH, Pages F, Sautes-Fridman C, et al. The immune contexture in human tumours: impact on clinical outcome. Nat Rev Cancer 2012;12:298-306.

14. Kilic A, Landreneau RJ, Luketich JD, et al. Density of tumor-infiltrating lymphocytes correlates with disease recurrence and survival in patients with large non-smallcell lung cancer tumors. J Surg Res 2011;167:207-10.

15. Travis WD, Brambilla E, Burke AP, et al. WHO Classification of Tumours of the Lung, Pleura, Thymus and Heart. 4th ed. Lyon: IARC Press; 2015.

16. Detterbeck FC, Chansky K, Groome P, et al. The IASLC Lung Cancer Staging Project: Methodology and Validation Used in the Development of Proposals for Revision of the Stage Classification of NSCLC in the Forthcoming (Eighth) Edition of the TNM Classification of Lung Cancer. J Thorac Oncol 2016;11:1433-46.

17. Tsao SM, Kerr KM. editors. IASLC ATLAS of PD-L1 immunohistochemistry testing in lung cancer. Aurora: Editorial RX Press, 2017.

18. Rakaee M, Kilvaer TK, Dalen SM, et al. Evaluation of tumor-infiltrating lymphocytes using routine H\&E slides predicts patient survival in resected non-small cell lung cancer. Hum Pathol 2018;79:188-98.

19. Midha A, Sharpe A, Scott M, et al. PD-L1 expression in advanced NSCLC: Primary lesions versus metastatic sites and impact of sample age. J Clin Oncol 2016;34:abstr 3025.

20. Grillo F, Bruzzone M, Pigozzi S, et al. Immunohistochemistry on old archival paraffin blocks: is there an expiry date? J Clin Pathol 2017;70:988-93.

21. Huynh TG, Morales-Oyarvide V, Campo MJ, et al. Programmed Cell Death Ligand 1 Expression in Resected Lung Adenocarcinomas: Association with Immune

Cite this article as: Jin Y, Shen X, Pan Y, Zheng Q, Chen H, $\mathrm{Hu} \mathrm{H}, \mathrm{Li}$ Y. Correlation between PD-L1 expression and clinicopathological characteristics of non-small cell lung cancer: A real-world study of a large Chinese cohort. J Thorac Dis 2019;11(11):4591-4601. doi: 10.21037/jtd.2019.10.80
Microenvironment. J Thorac Oncol 2016;11:1869-78.

22. Naso JR, Wang G, Pender A, et al. Intratumoral Heterogeneity in PD-L1 Immunoreactivity is Associated with Variation in Non-Small Cell Lung Carcinoma Histotype. Histopathology 2019. [Epub ahead of print].

23. Dietel M, Savelov N, Salanova R, et al. Real-world prevalence of programmed death ligand 1 expression in locally advanced or metastatic non-small-cell lung cancer: The global, multicenter EXPRESS study. Lung Cancer 2019;134:174-9.

24. Bassanelli M, Sioletic S, Martini M, et al. Heterogeneity of PD-L1 Expression and Relationship with Biology of NSCLC. Anticancer Res 2018;38:3789-96.

25. Munari E, Zamboni G, Lunardi G, et al. PD-L1 Expression Heterogeneity in Non-Small Cell Lung Cancer: Defining Criteria for Harmonization between Biopsy Specimens and Whole Sections. J Thorac Oncol 2018;13:1113-20.

26. Li C, Huang C, Mok TS, et al. Comparison of 22C3 PDL1 Expression between Surgically Resected Specimens and Paired Tissue Microarrays in Non-Small Cell Lung Cancer. J Thorac Oncol 2017;12:1536-43.

27. Büttner R, Gosney JR, Skov BG, et al. Programmed Death-Ligand 1 Immunohistochemistry Testing: A Review of Analytical Assays and Clinical Implementation in NonSmall-Cell Lung Cancer. J Clin Oncol 2017;35:3867-76.

28. Rangachari D, VanderLaan PA, Shea M, et al. Correlation between Classic Driver Oncogene Mutations in EGFR, ALK, or ROS1 and 22C3-PD-L1 >/=50\% Expression in Lung Adenocarcinoma. J Thorac Oncol 2017;12:878-83.

29. Tsao MS, Le Teuff G, Shepherd FA, et al. PD-L1 protein expression assessed by immunohistochemistry is neither prognostic nor predictive of benefit from adjuvant chemotherapy in resected non-small cell lung cancer. Ann Oncol 2017;28:882-9.

30. Toki MI, Mani N, Smithy JW, et al. Immune marker profiling and PD-L1 expression across Non-Small Cell Lung Cancer mutations. J Thorac Oncol 2018;13:1884-96. 